Review

\title{
Common Chromosomal Fragile Site Gene WWOX in Metabolic Disorders and Tumors
}

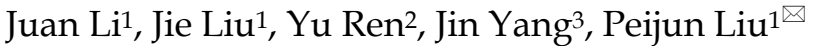 \\ 1. Center for Translational Medicine, The First Affiliated Hospital of Xian Jiaotong University College of Medicine; \\ 2. Department of Surgical Oncology, The First Affiliated Hospital of Xian Jiaotong University College of Medicine; \\ 3. Department of Oncology, The First Affiliated Hospital of Xian Jiaotong University College of Medicine.
}

$\triangle$ Corresponding author: Peijun Liu, Ph.D.\&M.D., Professor, Email: liupeijun@mail.xjtu.edu.cn, Tel: 086-18991232306, Address: 277 Yanta Western Rd., Xi'an, Shaan Xi Province, China, 710061.

() Ivyspring International Publisher. This is an open-access article distributed under the terms of the Creative Commons License (http://creativecommons.org/ licenses/by-nc-nd/3.0/). Reproduction is permitted for personal, noncommercial use, provided that the article is in whole, unmodified, and properly cited.

Received: 2013.09.22; Accepted: 2013.II.14; Published: 2014.01.II

\begin{abstract}
WWOX, a gene that spans the second most common chromosomal fragile site (FRAI6D), often exhibits homozygous deletions and translocation breakpoints under multiple cellular stresses induced by extrinsic or intrinsic factors, such as hypoxia, UV, and DNA damage regents. Loss of WWOX is closely related to genomic instability, tumorigenesis, cancer progression and therapy resistance. WWOX heterozygous knockout mice show an increased incidence of spontaneous or induced tumors. WWOX can interact via the WW domain with proteins that possess proline PPxY motifs and is involved in a variety of cellular processes. Accumulating evidence has shown that WWOX that contains a short-chain dehydrogenase/reductase (SDR) domain is involved in steroid metabolism and bone development. Reduced or lost expression of WWOX will lead to development of metabolic disease. In this review, we focus on the roles of WWOX in metabolic disorders and tumors.
\end{abstract}

Key words: WW domain-containing oxidoreductase; metabolic disorders; tumorigenesis; endocrine and chemotherapy.

\section{Introduction}

Fragile sites (FSs) are chromosome regions that exhibit an increased occurrence frequency of gaps or breaks when exposed to extrinsic or intrinsic DNA replication inhibitors such as BrdU, 5-azacytidine, aphidicolin and hypoxia [1, 2]. FSs are subdivided into common and rare fragile sites according to their occurrence frequency in the human population. Common fragile sites (CFS) are seen in all individuals, while rare fragile sites (RFS) are found in less than 5\% of the human population [3].

CFSs are components of normal chromosome structure and are not the result of the expansion of repeat sequences. FRA3B (3p14.2), FRA16D (16q23.2) and FRAXB (Xp21.1) are currently the most characteristic CFSs. FRA16D, the second highly expressed CFS, encompasses the WWOX gene encoding a WW domain-containing oxidoreductase. The WWOX gene spans a genomic locus of more than $1 \mathrm{Mbp}$ encoding an open reading frame of $1.2 \mathrm{~Kb}$, and consists of 9 coding exons in a region of approximately one million base pairs $[4,5]$.

The full-length WWOX, which encodes a 414 amino acid protein, possesses two typical N-terminal WW domains (first domain, amino acids 17-49; second domain, amino acids 58-90), a C-terminal short-chain dehydrogenase reductase (SDR) domain, and a nuclear localization sequence (NLS) (Fig.1). The first N-terminal WW domain is needed for the classical WW-PPXY interaction. The proteins with the SDR domain are involved in oxidation and reduction of various substrates such as lipid hormones, sugars, alcohols and retinoids [6]. 


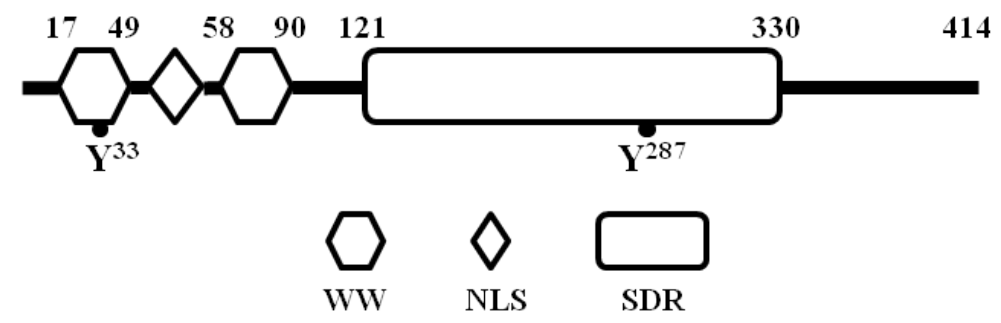

Figure I.Domains and phosphorylation of human WWOX protein.

Endogenous WWOX remains in mitochondria in cultured normal cell lines such as epidermal keratinocytes and mammary gland cells, and is translocated to nuclei under tumor necrosis factor- $\alpha$ (TNFa) stimulation [7, 8]. The subcellular localization and function of WWOX are regulated by its phosphorylation. WWOX Tyr33 phosphorylation can be stimulated by steriod hormone $17 \beta$-estradiol (E2) independent of the estrogen receptor (ER) [9]. WWOX can also be translocated into the nucleus upon Tyr33 phosphorylation induced by anisomycin or UV light at the first N-terminal WW domain of WWOX in L929 fibroblasts and other cell lines. Activated Cdc42-associated kinase (ACK1) can phosphorylate WWOX at Tyr33 and Tyr287, and the Tyr287 phosphorylation process is associated with WWOX polyubiquitination and degradation. However, the E3 ubiquitin ligase needed for WWOX ubiquitination has not been identified $[7,8,10]$.

Numerous studies have shown that WWOX is an important tumor suppressor and that loss or deregulation of WWOX contributes to development of various tumors. In addition, WWOX is involved in steroid hormone metabolism [11]. The focus of this review is to summarize the roles of WWOX in metabolic diseases and tumor development.

The wild-type WWOX protein contains 414 amino acids and possesses two N-terminal WW domains (first domain, amino acids 17-49; second domain, amino acids 58-90) and a C-terminal short-chain alcohol dehydrogenase reductase (SDR) domain. A nuclear localization sequence (NLS) is located between the two WW domains. $\operatorname{Tyr}(33)$ and $\operatorname{Tyr}(287)$ are the known sites of WWOX phosphorylation.

\section{WWOX dysregulation is associated with metabolic disorders}

\section{Steroid metabolism disorders}

The WWOX protein with a classcial SDR domain is associated with oxidation and reduction of lipid hormones. Meanwhile, WWOX has been found to be highly expressed in hormone-dependent tissues, such as the prostate, mammary gland and ovary, and secretory epithelial cells of endocrine and exocrine tissues [12]. The WWOX distribution patterns lead to the speculation that this protein is perhaps involved in regulation of the activities of metabolic steroids or steroid receptors.

The WWOX gene alternatively knocked out in mice is commonly used to study the roles of WWOX in steroid metabolism. WWOX deficient mice are born with gonad abnormalities, including failure of Leydig cell development in testis and reduction of theca cell proliferation in ovary. Loss of WWOX induces reduced levels of the testosterone and affects the normal functions of the prostate [11]. Knockout (KO) mice may suffer from impaired mammary branching morphogenesis due to the haploinsuffisiency of WWOX in the mouse mammary gland [13]. Thus, WWOX is essential for gonadal development and function.

WWOX $/$ - mice show reduced expressions of key steroidogenesis enzymes, including Cyp11a1 (cytochrome P450, family 11, subfamily A, polypeptide 1), Cyp17a1 (cytochrome P450, family 17, subfamily A, polypeptide 1$)$, and Hsd3b6 ( $3 \beta$-hydroxysteriod dehydrogenase). It has been reported that reduced expression of key steroidogenesis enzymes is the main reason for abnormal Leydig cells and decreased level of testosterone in WWOX ${ }^{-/}$mice [11]. Therefore, WWOX may regulate steroid metabolism by regulation of activities of key steroidogenesis enzymes.

\section{Bone metabolism disorders}

Mice with a homozygous deletion of WWOX show reduced serum calcium, hypoproteinuria, and hypoglycemia, leading to development of metabolic bone diseases $[14,15]$. WWOX $/-$ mice also suffer a disorder of skeleton development and increased osteoclast activity, characterized by thinner cortical bones and decreased mineral density and bone volume [16]. WWOX may affect the bone development via interaction with RUNX2 (Runt-related transcription factor 2). RUNX2 is a principal transcriptional regulator of osteoblast differentiation [14] and has been found to be responsible for metabolic bone abnormalities of WWOX $\%$ mice. Loss of WWOX ex- 
pression activates RUNX2-mediated transcription activity, enhances the expression levels of downstream target genes, including VEGF and osteocalcin $[17,18]$, and induces bone metabolic defects.

Taken together, reduced or lost expression of WWOX will lead to development of metabolic diseases due to the role of the SDR domain-containing protein WWOX in steroid metabolism and bone development.

\section{WWOX is a tumor suppressor}

\section{WWOX expressions are decreased in tumor tissues and cancer cells}

Loss or deregulation of WWOX expression is associated with the development of various cancer types and cancer cell lines, including pancreatic adenocarcinoma, renal cell carcinoma and endocrine and exocrine carcinomas $[19,20]$ (Tab.1).

Immunohistochemical staining has shown strong WWOX expression in the normal human ovarian tissue, but lack of or barely detectable WWOX expression in $37 \%$ of tumors [21]. Eighty-four percent of prostate cancers have down-modulated WWOX
[22]. Esophageal squamous cell carcinoma, non-small cell lung cancer and breast cancer show a high loss of heterozygosity $(\mathrm{LOH})$, a low mutation rate and the expression of aberrant transcripts of the WWOX gene. Northern blot and RT-PCR analyses of the breast and ovarian cancer cells have revealed that the presence of transcripts of smaller sizes represents abnormally spliced versions of WWOX [20]. DNA hypermethylation in the regulatory region has been found to be the cause of down-modulation of WWOX expression in prostate cancer-derived cells. The WWOX mRNA and protein expressions in prostate cancer cells can be increased by 5-aza-2-deoxycytidine (AZA), a DNA methytransferase inhibitor [22].

The WWOX expression is associated with the hormone receptor status. ER or PR positive cancer has a higher level of WWOX expression than ER or PR negative cancer [23]. Reduced WWOX expression demonstrates a significant correlation with the clinical stages of the cancer, negative ER status and poor survival. Thus, the expression status of WWOX has an effect on cancer progression and prognosis [21, 24].

Table I. Dysregulation of WWOX expression in various cancers.

\begin{tabular}{|c|c|c|c|}
\hline Material & Technique & Frequency & Ref \\
\hline Breast cancer (invasive) & Immunohistochemistry & $63.2 \%-60 \%$ (reduction) & {$[25,26]$} \\
\hline $\mathrm{ER}^{+}$breast carcinomas & Immunohistochemistry & $27 \%$ (negative) & {$[27,28]$} \\
\hline ER-breast carcinomas & Immunohistochemistry & $46 \%$ (negative) & \\
\hline Pancreatic cancer & RT-PCR/immunoblotting & $40 \%$ (reduction) & [29] \\
\hline Ovarian carcinoma & Immunohistochemistry/ immunoblotting & $37 \%$ (reduction) & {$[30]$} \\
\hline Prostate cancer & Immunohistochemistry & $84 \%$ (reduction) & {$[22]$} \\
\hline Osteosarcoma & Immunohistochemistry & $58 \%$ (reduction) & {$[18]$} \\
\hline Renal cell carcinoma & Immunohistochemistry/ immunoblotting/ RT-PCR & $60.7 \%$ (reduction) & [31] \\
\hline Oesophageal squamous & sequencing & $39 \%(\mathrm{LOH})$ & {$[32]$} \\
\hline Non-small cell lung cancer & Immunohistochemistry & $84.9 \%$ (reduction) & [33] \\
\hline Primary hematopoietic & RT-PCR/ sequencing & $51 \%$ (reduction) & {$[34]$} \\
\hline
\end{tabular}

\section{WWOX deregulation is associated with carcinogenesis and metastasis}

Reduced WWOX expression is associated with carcinogenesis and progression of various cancers. Heterozygous WWOX KO mice have increased incidence of spontaneous tumors. When mice are given a single dose of ENU (powerful chemical mutagen), $17.2 \%(10 / 58)$ of the heterozygous WWOX KO mice develop papillary carcinoma of the lung, compared with $3.3 \%(2 / 60)$ of the wide type [12]. Abnormal activation of Wnt/beta-Catenin signal pathway is closely related to tumorigenesis and tumor development [35]. Bouteille $\mathrm{N}$ et al, using the two-hybrid system and coimmunoprecipitation assay, have veri- fied that WWOX physically associates with Dishevelled (Dvl) proteins, which are involved in the Wnt/ beta-Catenin pathway, and inhibits the nuclear import of Dvl proteins. Inhibition of endogenous WWOX expression results in stimulation of the transcriptional activity of the Wnt/beta-Catenin pathway [36]. These findings reveal that WWOX functions as a tumor suppressor gene and plays a critical role in inhibition of tumor formation.

Exogenous WWOX expression suppresses cancer cell growth and induces apoptosis. Overexpression of WWOX in DU145/LNCaP/PC-3 (WWOX negative prostate cancer cell) significantly reduces the number of cancer cell colonies, leading to apoptosis through caspase-dependent mechanism [22, 37]. 
WWOX is known to be involved in cell cycle and apoptotic process by interacting with the proteins that posses PPxY motifs, including p73 (p53 homologue), ErbB4 (erythroblastic leukemiavial ongene homolog 4 ), AP- $2 \gamma$ (activator protein $2 \gamma$ ) and C-Jun (Fig.2). Transient overexpression experiments have shown that WWOX suppresses the transcriptional functions of these proteins by sequestering them in the cytoplasm of cells. It has been demonstrated that WWOX can physically interact via its first WW domain with p73. Src kinase phosphorylates WWOX at Y33 in the first WW domain and Src-mediated phosphorylation of WWOX enhances its binding to p73. Furthermore, expression of WWOX leads to sequestration of p73 from nuclear to cytoplasm and its cytoplastic colocalization with WWOX. Cytoplasmic p73 contributes to the apoptotic activity of WWOX [38-42]. WWOX physically interacts with mitogen-activated protein kinase 1 (MEK1), which is located in the lipid rafts of the cell membrane. Pro-apoptotic regent phorobol myristate acetate (PMA) induces dissociation of the MEK/ WWOX complex, releases WWOX to mitochondria and causes apoptosis in Jurkat $\mathrm{T}$ cells [43]. Thus, WWOX suppresses tumorigenicity through down-regulation of onco-proteins by protein-protein interaction.

WWOX has a critical role in cancer metastasis and invasion. WWOX protein expression is lost or reduced in nearly $100 \%$ of metastatic cancer tissues. Reduced protein level of WWOX increases migration of cancer cells through the basal membrane [44, 45]. Intergrin a 3 is a transmembrane receptor that mediates the attachment between cells and extracellular matrix (ECM) and plays a vital role in migration and tumor invasion. Loss of WWOX expression induces the membranous intergrin a 3 protein expression and modulates the interaction between cancer cells and ECM, resulting in migration of cancer cells through the basal membrane $[46,47]$.

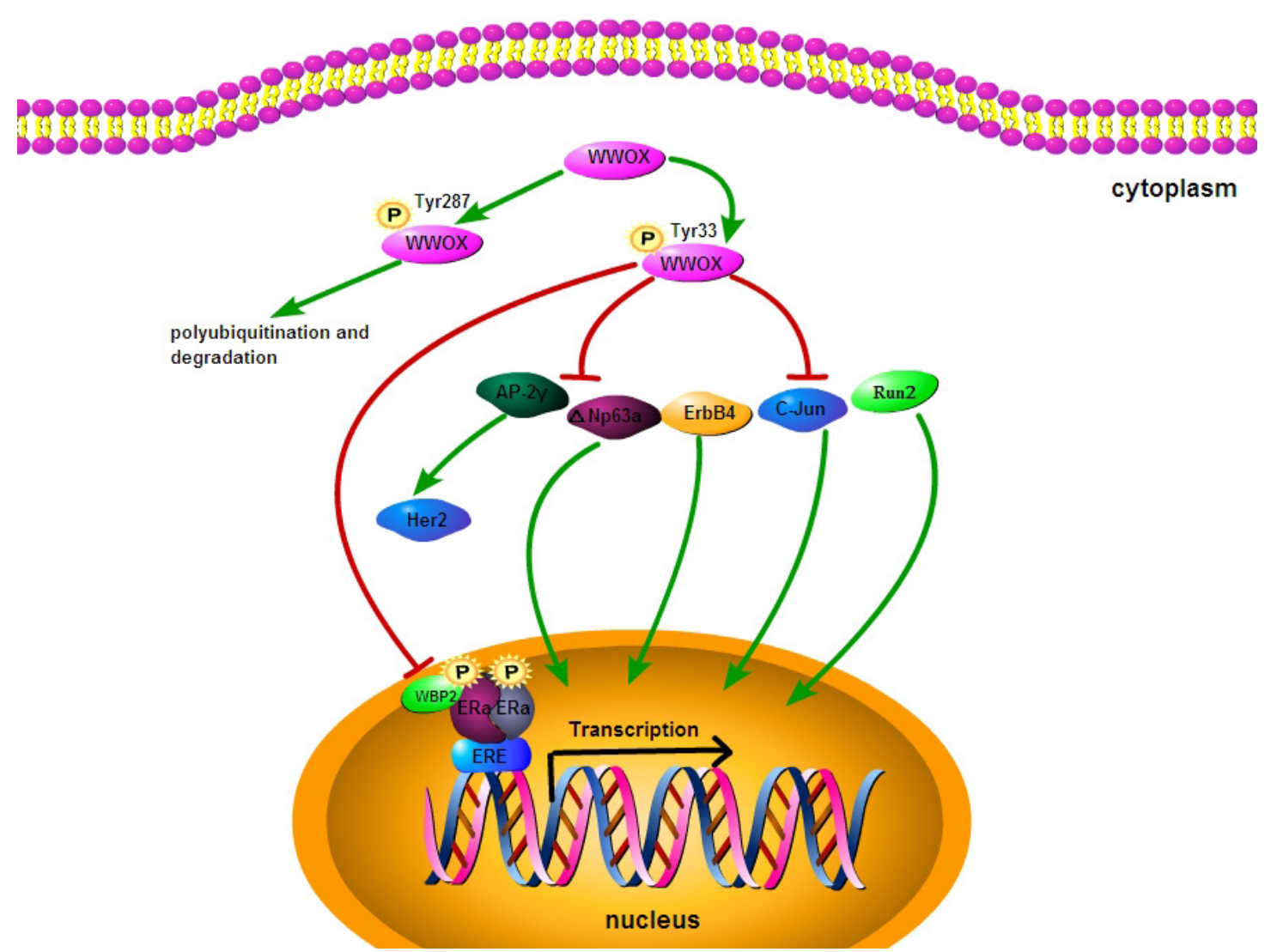

Figure 2. Regulatory mechanisms acting on WWOX.

\section{WWOX in endocrine therapy and chemotherapy for cancers}

Tamoxifen is the first choice as a selective estrogen receptor modulator (SERM) in clinical practice for endocrine treatment of early and advanced breast cancers. Tamoxifen functions as a competitive inhibitor of ER and represses the ER transcriptional activity [49]. To investigate the correlation of WWOX expression with the outcome of tamoxifen treatment, Gothlin EA et al have examined paraffin-embedded tissues from 912 randomized breast cancer patients. The 
WWOX protein expression level has been analyzed by using immunohistochemistry and statistic evaluation, and their results indicate that patients with high expression of WWOX may gain more benefit from tamoxifen treatment [48]. Research has found that the status of ER and PR in breast carcinomas is correlated with loss of WWOX expression. $49.2 \%$ of $\mathrm{ER}^{+}$breast carcinomas and $26.6 \%$ of ER- cases are strongly positive for WWOX expression. $26.2 \%$ of $\mathrm{ER}^{+} \mathrm{PR}^{+}$breast carcinomas and $50.9 \%$ of ER-PR- cases are completely negative for WWOX expression [50]. The strong association of WWOX expression with ER status suggests that WWOX may affect tamoxifen endocrine therapy for breast cancer. However, the underlying mechanism for the regulation of the association between WWOX and ER status is not clear. The WW domain binding protein-2 (WBP2) may be a key regulatory protein. As a coactivator for ER and PR transactivation pathways, WBP2 physically interacts with WWOX and activates steroid hormone receptors [51]. It has been reported that WWOX binds to the classic WW-PPXY motif of WBP2 [52], and suppresses transactivation pathways by interaction with WBP2. Loss of WWOX expression may up-regulate ER transcriptional activity and induces tamoxifen resistance. In addition, loss of WWOX expression induces the release of Ap2 $\gamma$ transcription factor protein to the nucleus and the up-regulation of epidermal receptor-2 (Her2). Increased Her2 enhances the agonistic activity against endocrine therapy for breast cancer, indicating that WWOX may mediate tamoxifen resistance via interaction with Ap2 $\gamma$ [50, 53] (Fig.2). Thus, Loss of WWOX expression may increase tamoxifen resistance by up-regulating ER and Her2 transcriptional activities.

Cisplatin is among the most widely used chemotherapeutic agent for cancers. $\Delta N p 63 a$, a transcription factor localized in the nucleus, plays a crucial role in determining cellular sensitivity to chemotherapeutic agent [54,55]. It has been found that treatment of WWOX/ $\Delta$ Np63a-expressing cancer cells with cisplatin can increase the cell death by 14 folds, as compared with treatment of $\Delta \mathrm{Np} 63$ expressing cells alone. The WWOX- $\triangle$ Np63a interaction leads to increased chemosenstivity to cisplatin and an increased cell death rate. The underlying mechanism is probably that WWOX suppresses the transcriptional ability of $\triangle \mathrm{Np} 63$ by sequestering it in the cytoplasm [42]. Thus, WWOX may help maintain the sensitivity of cancer cells to chemotherapeutic drugs.

The above literature review shows that WWOX, as a chromosomal fragile site gene, can increase the sensitivity to endocrine therapy and chemotherapy for tumors.
WWOX is involved in cell cycle and apoptotic process by interacting with the proteins that posses PPxY motifs, including $\triangle \mathrm{Np} 63 \mathrm{a}$, ErbB4, AP-2 $\gamma$, Run2 and C-Jun. WWOX can sequester them in the cytoplasm and suppress their transcriptional functions. WWOX binds to the classic WW-PPXY motif of WBP2 and may down-regulate ER and PR transactivation pathways by suppressing the activity of coactivator WBP2. Tyr287 phosphorylation process is associated with WWOX polyubiquitination and degradation.

\section{Conclusions and future directions}

CFS gene WWOX has a critical role in steroid and bone metabolism. The WWOX protein is associated with oxidation and reduction of lipid hormones since it contains the classcial SDR domain. WWOX is highly expressed in hormone-dependent tissues and perhaps involved in regulation of activities of metabolic steroids. WWOX can enhance the activities of key steroidogenesis enzymes transcriptional activity of RUN2. Reduced or lost expression of WWOX will lead to development of metabolic diseases. Loss or dysregulation of WWOX expression also leads to genomic instability, tumorigenesis, cancer progression and resistance to therapy. WWOX has been shown to be involved in pro-apoptotic progression. WWOX may affect tumorigenesis by inhibiting activities of some proteins, including p73, ErbB4, AP- $2 \gamma$ and other transcription factors. Reduced WWOX expression is significantly correlated with clinical stages, negativity for hormone receptors and poor survival. Therefore, WWOX can be used as a marker for poor prognosis or unfavorable clinical outcomes.

Nevertheless, the function of WWOX SDR domain is still unclear. Is WWOX SDR domain related to the functions of hormone receptors and endocrine therapy resistance? Is WWOX dehydrogenase/reductase activity related to its tumor suppressive function? Answers to these questions will help to further understand the role of WWOX in metabolic diseases and tumors. Another future research direction may be to develop a tissue-specific (for example: hormone-related tissue) conditional knockout mouse model for further analysis of the role of WWOX in tissue development and metabolic diseases and for description of the biological functions of the SDR domain in vivo. In addition, the signal network of WWOX is still unclear. Further research is needed to elucidate the signaling network to shed light upon the role of WWOX in metabolic diseases and tumors. 


\section{Acknowledgement}

This work was supported by the First Affiliated Hospital of Xian Jiaotong University College of Medicine Foundation grant 2011 YK.16.

\section{Competing Interests}

The authors have declared that no competing interest exists.

\section{References}

1. Dillon LW, Burrow AA, Wang YH. DNA instability at chromosomal fragile sites in cancer. Current genomics. 2010; 11: 326-37.

2. Lukusa T, Fryns JP. Human chromosome fragility. Biochimica et biophysica acta. 2008 ; 1779: 3-16.

3. Durkin SG, Glover TW. Chromosome fragile sites. Annual review of genetics. 2007; 41: 169-92.

4. Popescu NC. Genetic alterations in cancer as a result of breakage at fragile sites. Cancer letters. 2003; 192: 1-17.

5. Smith DI, McAvoy S, Zhu Y, Perez DS. Large common fragile site genes and cancer. Seminars in cancer biology. 2007; 17: 31-41.

6. Chang NS, Hsu LJ, Lin YS, Lai FJ, Sheu HM. WW domain-containing oxidoreductase: a candidate tumor suppressor. Trends in molecular medicine. 2007; 13: 12-22.

7. Watanabe A, Hippo Y, Taniguchi H, Iwanari H, Yashiro M, Hirakawa K, et al. An opposing view on WWOX protein function as a tumor suppressor. Cancer research. 2003; 63: 8629-33.

8. Mahajan K, Mahajan NP. Shepherding AKT and androgen receptor by Ack1 tyrosine kinase. Journal of cellular physiology. 2010; 224: 327-33.

9. Chang NS, Schultz L, Hsu LJ, Lewis J, Su M, Sze CI. 17beta-Estradiol upregulates and activates WOX1/WWOXv1 and WOX2/WWOXv2 in vitro: potential role in cancerous progression of breast and prostate to a premetastatic state in vivo. Oncogene. 2005; 24: 714-23.

10. Mahajan NP, Whang YE, Mohler JL, Earp HS. Activated tyrosine kinase Ack1 promotes prostate tumorigenesis: role of Ack1 in polyubiquitination of tumor suppressor Wwox. Cancer research. 2005; 65: 10514-23.

11. Aqeilan RI, Hagan JP, de Bruin A, Rawahneh M, Salah Z, Gaudio E, et al. Targeted ablation of the WW domain-containing oxidoreductase tumor suppressor leads to impaired steroidogenesis. Endocrinology. 2009; 150: 1530-5.

12. Aqeilan RI, Trapasso F, Hussain S, Costinean S, Marshall D, Pekarsky Y, et al. Targeted deletion of Wwox reveals a tumor suppressor function. Proceedings of the National Academy of Sciences of the United States of America. 2007; 104: 3949-54.

13. Ferguson BW, Gao X, Kil H, Lee J, Benavides F, Abba MC, et al. Conditional Wwox deletion in mouse mammary gland by means of two Cre recombinase approaches. PloS one. 2012; 7: e36618.

14. Aqeilan RI, Hassan MQ de Bruin A, Hagan JP, Volinia S, Palumbo T, et al. The WWOX tumor suppressor is essential for postnatal survival and normal bone metabolism. The Journal of biological chemistry. 2008; 283: 21629-39.

15. Ludes-Meyers JH, Kil H, Parker-Thornburg J, Kusewitt DF, Bedford MT, Aldaz CM. Generation and characterization of mice carrying a conditional allele of the Wwox tumor suppressor gene. PloS one. 2009; 4: e7775.

16. Suzuki H, Katayama K, Takenaka M, Amakasu K, Saito K, Suzuki K. A spontaneous mutation of the Wwox gene and audiogenic seizures in rats with lethal dwarfism and epilepsy. Genes, brain, and behavior. 2009; 8: 650-60.

17. Del Mare S, Kurek KC, Stein GS, Lian JB, Aqeilan RI. Role of the WWOX tumor suppressor gene in bone homeostasis and the pathogenesis of osteosarcoma. American journal of cancer research. 2011; 1: 585-94.

18. Kurek KC, Del Mare S, Salah Z, Abdeen S, Sadiq H, Lee SH, et al. Frequent attenuation of the WWOX tumor suppressor in osteosarcoma is associated with increased tumorigenicity and aberrant RUNX2 expression. Cancer research. 2010; 70: 5577-86

19. Lin JT, Tzai TS, Liao CY, Wang JS, Wu TT, Wang HY, et al. WWOX protein expression varies among RCC histotypes and downregulation of WWOX protein correlates with less-favorable prognosis in clear RCC. Annals of surgical oncology. 2013; 20: 193-9.

20. Iliopoulos D, Guler G, Han SY, Druck T, Ottey M, McCorkell KA, et al. Roles of FHIT and WWOX fragile genes in cancer. Cancer letters. 2006; 232: 27-36.

21. Nunez MI, Rosen DG, Ludes-Meyers JH, Abba MC, Kil H, Page R, et al. WWOX protein expression varies among ovarian carcinoma histotypes and correlates with less favorable outcome. BMC cancer. 2005:5:64.

22. Qin HR, Iliopoulos D, Semba S, Fabbri M, Druck T, Volinia S, et al. A role for the WWOX gene in prostate cancer. Cancer research. 2006; 66: 6477-81.

23. Pluciennik E, Kusinska R, Potemski P, Kubiak R, Kordek R, Bednarek AK. WWOX--the FRA16D cancer gene: expression correlation with breast cancer progression and prognosis. European journal of surgical oncology : the journal of the European Society of Surgical Oncology and the British Association of Surgical Oncology. 2006; 32: 153-7.
24. Paige AJ, Zucknick M, Janczar S, Paul J, Mein CA, Taylor KJ, et al. WWOX tumour suppressor gene polymorphisms and ovarian cancer pathology and prognosis. Eur J Cancer. 2010; 46: 818-25.

25. Guler G, Uner A, Guler N, Han S-Y, Iliopoulos D, Hauck WW, et al. The fragile genesFHIT andWWOX are inactivated coordinately in invasive breast carcinoma. Cancer. 2004; 100: 1605-14.

26. Płuciennik E, Kusińska R, Potemski P, Kubiak R, Kordek R, Bednarek AK. WWOX - the FRA16D cancer gene: Expression correlation with breast cancer progression and prognosis. European Journal of Surgical Oncology. 2006; 32: $153-7$

27. Nunez1 MaI. Frequent loss of WWOX expression in breast cancer: correlation with estrogen receptor status. Breast cancer research and treatment. 2005; 89: 99-105.

28. Nunez MI, Ludes-Meyers J, Aldaz CM. WWOX protein expression in normal human tissues. Journal of molecular histology. 2006; 37: 115-25.

29. Kuroki T. The Tumor Suppressor Gene WWOX at FRA16D Is Involved in Pancreatic Carcinogenesis. Clinical Cancer Research. 2004; 10: 2459-65.

30. Nunez MI, Rosen DG, Ludes-Meyers JH, Abba MC, Kil H, Page R, et al. WWOX protein expression varies among ovarian carcinoma histotypes and correlates with less favorable outcome. BMC Cancer. 2005; 5: 64

31. Lin JT, Tzai TS, Liao CY, Wang JS, Wu TT, Wang HY, et al. WWOX Protein Expression Varies Among RCC Histotypes and Downregulation of WWOX Protein Correlates with Less-Favorable Prognosis in Clear RCC. Ann Surg Oncol. 2012;20:193-9

32. Kuroki T TF, Shiraishi T, Alder H, Mimori K, Mori M, Croce CM. Genetic alterations of the tumor suppressor gene WWOX in esophageal squamous cell carcinoma. Cancer Res. 2002; 15: 2258-60.

33. Yendamuri S KT, Trapasso F, Henry AC, Dumon KR, Huebner K, Williams $\mathrm{NN}$, Kaiser LR, Croce CM. WW domain containing oxidoreductase gene expression is altered in non-small cell lung cancer. Cancer research. 2003; 15: 878-81.

34. Ishii H, Mimori K, Vecchione A, Sutheesophon K, Fujiwara T, Mori M, et al. Effect of exogenous E2F-1 on the expression of common chromosome fragile site genes, FHIT and WWOX. Biochemical and biophysical research communications. 2004; 316: 1088-93.

35. Taketo MM. Shutting down Wnt signal-activated cancer. NATURE GENETICS. 2004; 36: 320-2.

36. Bouteille N, Driouch $\mathrm{K}$, El Hage $\mathrm{P}$, Sin $\mathrm{S}$, Formstecher $\mathrm{E}$, Camonis J, et al. Inhibition of the Wnt/ $\beta$-catenin pathway by the WWOX tumor suppressor protein. Oncogene. 2009; 28: 2569-80.

37. Kosla K, Pluciennik E, Kurzyk A, Jesionek-Kupnicka D, Kordek R, Potemski P, et al. Molecular analysis of WWOX expression correlation with proliferation and apoptosis in glioblastoma multiforme. Journal of neuro-oncology. 2011; 101: 207-13.

38. Aqeilan RI, Donati V, Gaudio E, Nicoloso MS, Sundvall M, Korhonen A, et al. Association of Wwox with ErbB4 in breast cancer. Cancer research. 2007; 67: 9330-6.

39. Aqeilan RI, Palamarchuk A, Weigel RJ, Herrero JJ, Pekarsky Y, Croce CM. Physical and functional interactions between the Wwox tumor suppressor protein and the AP-2gamma transcription factor. Cancer research. 2004; 64: 8256-61.

40. Aqeilan RI, Pekarsky Y, Herrero JJ, Palamarchuk A, Letofsky J, Druck T, et al Functional association between Wwox tumor suppressor protein and p73, a p53 homolog. Proceedings of the National Academy of Sciences of the United States of America. 2004; 101: 4401-6.

41. Gaudio E, Palamarchuk A, Palumbo T, Trapasso F, Pekarsky Y, Croce CM, et al. Physical association with WWOX suppresses c-Jun transcriptional activity. Cancer research. 2006; 66: 11585-9.

42. Salah Z, Bar-mag T, Kohn Y, Pichiorri F, Palumbo T, Melino G, et al. Tumor suppressor WWOX binds to DeltaNp63alpha and sensitizes cancer cells to chemotherapy. Cell death \& disease. 2013; 4: e480.

43. Lin HP, Chang JY, Lin SR, Lee MH, Huang SS, Hsu LJ, et al. Identification of an In Vivo MEK/WOX1 Complex as a Master Switch for Apoptosis in T Cell Leukemia. Genes \& cancer. 2011; 2: 550-62.

44. Guler G, Uner A, Guler N, Han SY, Iliopoulos D, Hauck WW, et al. The fragile genes FHIT and WWOX are inactivated coordinately in invasive breast carcinoma. Cancer. 2004; 100: 1605-14.

45. Guler G, Himmetoglu C, Jimenez RE, Geyer SM, Wang WP, Costinean S, et al. Aberrant expression of DNA damage response proteins is associated with breast cancer subtype and clinical features. Breast cancer research and treatment. 2011; 129: 421-32.

46. Gourley C, Paige AJ, Taylor KJ, Ward C, Kuske B, Zhang J, et al. WWOX gene expression abolishes ovarian cancer tumorigenicity in vivo and decreases attachment to fibronectin via integrin alpha3. Cancer research. 2009; 69: 4835-42.

47. Zhang JQ, Li L, Song HL, Paige A, Gabra H. Effect of WWOX gene on the attachment and adhesion of ovarian cancer cells. Zhonghua fu chan ke za zhi. 2009; 44: 529-32.

48. Göthlin Eremo A, Wegman P, Stål O, Nordenskjöld B, Fornander T, Wingren S. Wwox expression may predict benefit from adjuvant tamoxifen in randomized breast cancer patients. Oncology reports. 2013; 29: 1467-74.

49. Orlandoa L., Schiavonea P., Fedelea P., et al. Molecularly targeted endocrine therapies for breast cancer. Cancer Treatment Reviews. 2010; 36S3:67-71. 
50. Nunez MI, Ludes-Meyers J, Abba MC, Kil H, Abbey NW, Page RE, et al. Frequent loss of WWOX expression in breast cancer: correlation with estrogen receptor status. Breast cancer research and treatment. 2005; 89: 99-105.

51. Dhananjayan SC, Ramamoorthy S, Khan OY, Ismail A, Sun J, Slingerland J, et al. WW domain binding protein-2, an E6-associated protein interacting protein, acts as a coactivator of estrogen and progesterone receptors. Mol Endocrinol. 2006; 20: 2343-54.

52. McDonald CB, Buffa L, Bar-Mag T, Salah Z, Bhat V, Mikles DC, et al. Biophysical basis of the binding of WWOX tumor suppressor to WBP1 and WBP2 adaptors. Journal of molecular biology. 2012; 422: 58-74

53. Guler G, Iliopoulos D, Guler N, Himmetoglu C, Hayran M, Huebner K. Wwox and Ap2gamma expression levels predict tamoxifen response. Clinical cancer research : an official journal of the American Association for Cancer Research. 2007; 13: 6115-21.

54. Zangen R, Ratovitski E, Sidransky D. $\Delta$ Np63a Leves Correlate with Clinical Tumor Response to Cisplatin. Cell cycle. 2005; 4: 1313-15.

55. Sen $T$, Sen $N$, Brait $M$, Begum $S$, Chatterjee $A$, Hoque $M O$, et al. DeltaNp63alpha confers tumor cell resistance to cisplatin through the AKT1 transcriptional regulation. Cancer research. 2011; 71: 1167-76. 